\title{
TOPOLOGICAL TRANSFORMATION GROUPS WITH A FIXED END POINT
}

\author{
WILLIAM J. GRAY
}

1. Let $(X, T, \pi)$ be a topological transformation group, where $X$ is a nontrivial Hausdorff space and $T$ is a topological group which leaves an end point $e$ of $X$ fixed. In [3], Wallace proved that if $T$ is cyclic and $X$ is a locally connected continuum, $T$ has a fixed point other than $e$. Then in [4], Wallace asked the following question: If $X$ is a peano continuum and $T$ is compact or abelian, then does $T$ have a fixed point other than $e$ ?

In [5] Wang showed that if $T$ is compact, and $X$ is arcwise connected, then $T$ has a fixed point other than $e$. Then Chu [1] showed that $T$ has infinitely many fixed points. Chu began the study of the abelian case in [2].

In this paper we show by example that $X$ may be a peano continuum and $T$ may be a countably generated abelian group which has $e$ for its only fixed point, $\S 2$. Thus in general the answer to Wallace's question in the abelian case is no. However, if $T$ is a generative group, and $X$ is compact and arcwise connected, then $T$ has a fixed point other than $e, \S 3$. We also show by example that $T$ may be a finitely generated nonabelian group which has $e$ for its only fixed point, $\$ 4$.

2. Let $\left\{P_{n} ; n \geqq 1\right\}$ be a sequence of points which lie on a line; $P_{n+1}$ lies to the right of $P_{n}$ and the distance from $P_{n+1}$ to $P_{n}$ is $1 / 2^{n}$. The limit of the $\left\{P_{n}\right\}$ is denoted by $e$. Construct a sequence of sets $\left\{X_{n} ; n \geqq 1\right\}$ as follows: $X_{1}, X_{2}$, and $X_{3}$ are shown in Figure 1 . In general, if $n>1, X_{n}=A_{n} \cup B_{n}$, where $A_{n}$ is the union of $X_{n-1}$ and the line segment from $P_{n-1}$ to $P_{n}$, and $B_{n}$ is a simplicial replica of $A_{n}$; we require that $B_{n}$ lies in a square, one of whose sides is the line segment from $P_{n-1}$ to $P_{n}$, and the intersection of $B_{n}$ and $A_{n}$ is exactly $P_{n}$. The peano continuum $X$ is the union of $e$ and all the $X_{n}$, where $X$ has the usual topology of the plane. A vertex of $X$ is a point other than $e$ where the space "branches." $X$ contains countably many simplicial replicas $\left\{X_{n}^{(\boldsymbol{k})}\right\}$ of $X_{n}$ for each $n$. These complexes contain $2^{n+1}-1$ vertices and are of the form $X_{n}^{(\boldsymbol{k})}=A_{n}^{(\boldsymbol{k})} \cup B_{n}^{(\boldsymbol{k})}$ where $A_{n}^{(\boldsymbol{k})}$ and $B_{n}^{(\boldsymbol{k})}$ are simplicial replicas of $A_{n}$ and $B_{n}$, respectively. $f_{n}$ is the homeomorphism: $X \rightarrow X$ which permutes $A_{n}^{(\boldsymbol{k})}$ and $B_{n}^{(k)}, k \geqq 1$, simplicially in a

Received by the editors August 17, 1965. 


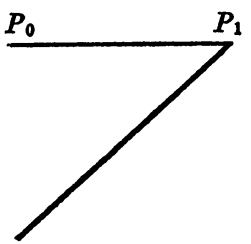

(a) $X_{1}$

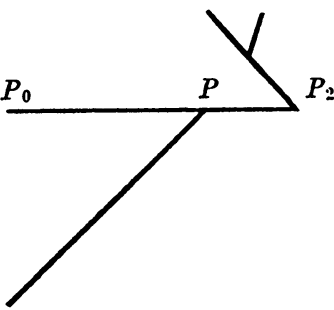

(b) $X_{2}$

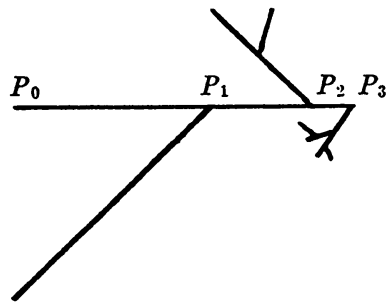

(c) $X_{3}$

FIGURE 1

natural fashion, and which leaves all other points of $X$, including $e$ fixed.

The fact that the elements of the sequence $\left\{f_{n}\right\}$ commute with each other is a consequence of the following facts:

(1) If $i<j, f_{i}\left(X_{j}\right) \subset X_{j}$.

(2) For every $i \geqq 1, f_{i}^{2}=$ identity.

(3) If $i<j$, and if $h_{1}$ and $h_{2}$ are two vertices which are permuted by $f_{i}$, then the vertices $f_{j} h_{1}$ and $f_{j} h_{2}$ are also permuted by $f_{i}$.

All the fixed points of $f_{n}$ lie to the right of $P_{n}$ for each $n$. Hence the $f_{i}$ can have no fixed point, other than $e$, in common.

If $T$ denotes the discrete abelian group generated by the $\left\{f_{n}\right\}$, then $T$ has $e$ for its only fixed point.

3. Theorem 1 below is a restatement of a result of Wallace [3] and Theorem 2 is equivalent to a result of Wang [5].

Theorem 1. Let $X$ be a nontrivial Hausdorff continuum and $t: X \rightarrow X$ be a homeomorphism which leaves an end point e of $X$ fixed. Let $A$ and $B$ be subcontinua of $X$ such that $A \cap B=\{z\}$, where $z \neq e, e \in A$, and $X$ $=A \cup B$. If there exists $y \in B$ such that $\left\{y, t^{-1} y\right\} \subset B$, either $t z=z$, or else one of the following must hold:

(a) For each integer $n \geqq 0, t^{n-1} A \subset t^{n} A$ and $t^{n} B \subset t^{n-1} B$. Set

$$
K=\mathrm{Cl}\left(\bigcup\left\{t^{n} A ; n \geqq 0\right\}\right), \quad L=\bigcap\left\{t^{n} B ; n \geqq 0\right\} .
$$

Then $K$ and $L$ are t-invariant continua and $X=K \cup L$.

(b) For each integer $n \geqq 0, t^{-(n-1)} A \subset t^{-n} A$ and $t^{-n} B \subset t^{-(n-1)} B$. Set

$$
K=\operatorname{Cl}\left(\bigcup\left\{t^{-n} A ; n \geqq 0\right\}\right), \quad L=\cap\left\{t^{-n} B ; n \geqq 0\right\} .
$$

Then $K$ and $L$ are $t$-invariant continua and $X=K \cup L$.

Theorem 2. Let $(X, T, \pi)$ be a topological transformation group, where $X$ is an arcwise connected Hausdorff space and $T$ leaves an end 
point e of $X$ fixed. Then if there is a nonempty $T$-invariant closed subset of $X$ which does not contain $e, T$ has a fixed point other than $e$.

LEMMA 1. Let $X$ be a nontrivial arcwise connected compact Hausdorff space and $t_{1}, \cdots, t_{n}$ be commuting homeomorphisms: $X \rightarrow X$. Then if each of the $t_{i}$ leaves an end point $e$ of $X$ fixed, then the $t_{i}$ have another fixed point in common.

Proof. The proof for the case $n=1$ is obtained by combining Theorems 1 and 2, see Chu [2]. We proceed by induction. Assume the theorem is true for $n=k$, with $k \geqq 1$. Let $z_{j}$ be a fixed point common to $t_{1}, \cdots, t_{j-1}, t_{j+1}, \cdots, t_{k+1}$, where $z_{j} \neq e$ and $1 \leqq j \leqq k+1$. Set $A=\left\{z_{1}, \cdots, z_{k+1}\right\}$. Since $e$ is an end point and $e \notin A$, we may find subcontinua $A_{1}$ and $B_{1}$ of $X$ for which $X=A_{1} \cup B_{1}, e \in A_{1}, A \subset B_{1}$, $A_{1} \cap B_{1}=\{x\}$, where $x \neq e$. If $t_{i} x=x$ for $i=1, \cdots, k+1$, we are through. Otherwise we may assume without loss of generality that $t_{1} x \neq x$. Thus $t_{1}, A_{1}$, and $B_{1}$ satisfy the hypothesis of Theorem 1 , and we may assume that part (a) of Theorem 1 is applicable. If

$$
B_{2}=\bigcap\left\{t_{1}^{r} B_{1} ; r \geqq 0\right\},
$$

then $B_{2}$ is $t_{1}$-invariant and

$$
t_{1} B_{1} \subset t_{1}^{r-1} B_{1}, \quad r \geqq 1 .
$$

Now $z_{1} \in B_{1}$, and therefore $t_{1}^{r} z_{1} \in t_{1}^{r} B_{1}$; the sequence $\left\{t_{1}^{r} z_{1} ; r \geqq 0\right\}$ has a cluster point $w \in X$. Because of (1), it is clear that we may take $w \in B_{2}$. For each $i>1$, we have $t_{i} t_{1}^{r} z_{1}=t_{1}^{r} t_{i} z_{1}=t_{1}^{r} z_{1}$ so that $t_{i} w=w, i>1$. By recursion, define

$$
B_{j}=\bigcap\left\{t_{j-1}^{r} B_{j-1} ; r=0, \pm 1, \cdots\right\},
$$

for $j=1, \cdots, k+2$. Then $B_{j}$ is invariant under $t_{1}, \cdots, t_{j-1}$, and $w \in B_{j}$. Thus $B_{k+2}$ is a closed, nonempty subset of $X$ which is invariant under $t_{1}, \cdots, t_{k+1}$, and $e \notin B_{k+2}$ since

$$
B_{1} \supset B_{2} \supset \cdots \supset B_{k+2} \text {. }
$$

By Theorem 2, the proof is complete.

The example of $\$ 4$ shows that Lemma 1 is not true if the $t_{i}$ do not commute.

Lemma 2. Let $(X, T, \pi)$ be a topological transformation group, where $X$ satisfies the hypothesis of Lemma 1 and $T \simeq Z^{n} R^{m}$, where $Z$ is the additive group of all integers with the discrete topology and $R$ is the additive 


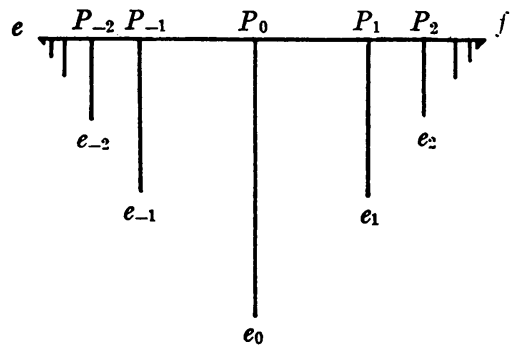

(a) $X_{1}$

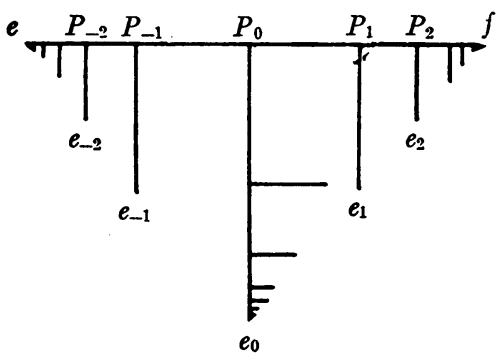

(b) $X_{2}$

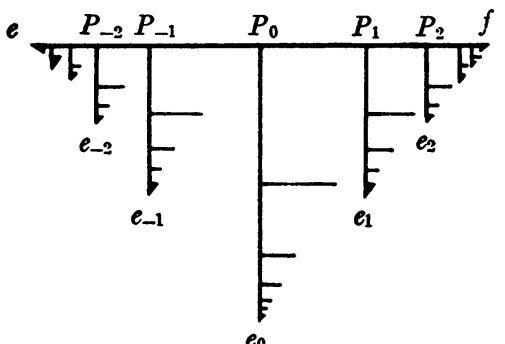

(c) $X_{3}$

FIGURE 2

group of all real numbers with the usual topology, and $m$ and $n$ are nonnegative integers. Then if $T$ leaves an end point $e$ of $X$ fixed, $T$ has a fixed point other than $e$.

Proof. There is a subgroup $A$ of $T$ such that $A \simeq Z^{n} \times Z^{m}$ and a compact subset $K \subset T$ for which $T=K A$. By Lemma 1 , there is a point $x \in X$, with $x \neq e$, which is fixed under $A$. Then $T x=K A x=K x$ is closed since $K$ is compact. Furthermore $e \notin T x$. By Theorem 2, the proof is complete. 
Theorem 3. Let $(X, T, \pi)$ be a transformation group, where $X$ satisfies the hypothesis of Lemma 1, and $T$ is a generative group (i.e., $T$ is generated by a compact neighborhood of the identity). If $T$ leaves an end point $e$ of $X$ fixed, $T$ has another fixed point.

The proof follows from Lemma 2 and Theorem 2 since we may write $T \simeq K Z^{n} R^{m}$, with $K$ compact, $Z$ and $R$ as in Lemma 2, see Chu [2].

4. Let $\left\{P_{k} ; k\right.$ an integer $\}$ be a sequence of points which lie on a line. $P_{k}$ lies to the left of $P_{k+1}$ and the distance from $P_{k+1}$ to $P_{k}$ is $1 / 2^{|k|-1}$ for $k \neq 0$. Let $e$ and $f$ be the limits of the sequences $\left\{P_{k} ; k<0\right\}$ and $\left\{P_{k} ; k>0\right\}$ respectively. Define a sequence $\left\{X_{n} ; n \geqq 1\right\}$ of spaces as follows: $X_{1}, X_{2}$, and $X_{3}$ are as in Figure 2. In general, in $X_{2 n}$, that portion of $X_{2 n}$ which lies on the line segment from $P_{0}$ to $e_{0}$ is a simplicial replica of that part of $X$ which lies on the segment from $P_{0}$ to $f$. In $X_{2 n+1}$ the part of the space on the segment from $P_{k}$ to $e_{k}$ is a replica of the portion of the space which lies on the segment from $P_{k+1}$ to $e_{k+1}$. The peano continuum $X$ is the union of all the $X_{n}$, where $X$ has the topology induced by the plane.

Define homeomorphisms $t, s: X \rightarrow X$ as follows: $t(e)=e, t(f)=f$. Let $Y_{k}$ be the portion of $X$ which is the union of the segment from $P_{k}$ to $P_{k+1}$ and the part of $X$ which lies on the segment from $P_{k}$ to $e_{k}$. Then $Y_{k}$ and $Y_{k+1}$ are homeomorphic. Let $t$ "slide" $Y_{k}$ in to $Y_{k+1}$ for each in teger $k$. This defines $t$. Now the part of $X$ which lies on the segment from $P_{0}$ to $f$ is homeomorphic to the part lying on the segment from $P_{0}$ to $e_{0} . s$ permutes these two subspaces and leaves all other points of $X$ fixed. The only fixed points of $t$ are $e$ and $f$, and $s(f)=e_{0}$. Hence the discrete group $T$ generated by $s$ and $t$ has $e$ for its only fixed point. The fact that $T$ is not abelian follows from $\S 3$.

\section{REFERENCES}

1. Hsin Chu, $A$ note on transformation groups with a fixed end point, Proc. Amer. Math. Soc. (to appear).

2. - Fixed points in a transformation group, Pacific J. Math. (to appear).

3. A. D. Wallace, $A$ fixed point theorem, Bull. Amer. Math. Soc. 51 (1945), 413-416.

4. - Group invariant continua, Fund. Math. 36 (1949), 119-124.

5. H. C. Wang, A remark on transformation groups leaving fixed an end point, Proc. Amer. Math. Soc. 3 (1952), 548-549.

6. W. H. Gottschalk and G. A. Hedlund, Topological dynamics, Amer. Math. Soc. Colloq. Publ., Vol 36, Amer. Math. Soc., Providence, R. I., 1955.

University of Alabama 